\title{
POSITIVE POINTS IN POLAR LATTICES
}

\author{
F. HOSSAIN and R. T. WORLEY
}

(Received 15 July 1976, revised 17 July 1977)

Communicated by Jane Pitman

\begin{abstract}
The authors investigate

$\max \min \mu F(x) F(y)$

for two standard distance functions $F$ in $R^{2}$, where $\mu$ denotes the area of $\left\{x \in R^{2} ; F(x) \leqslant 1\right\}$, the maximum is over all (geometric) lattices $\Lambda$ in $R^{2}$ and the minimum is over all positive points $x \in \Lambda$ and $y \in \Lambda^{*}$ (the polar lattice of $\Lambda$ ). An application is given to a problem on fractional parts.
\end{abstract}

Subject classification (Amer. Math. Soc. (MOS) 1970): 10 E 05.

Let $\Lambda$ be a (geometric) lattice in $R^{n}$ and let

$$
\Lambda^{*}=\left\{y \in R^{n}: y \cdot x \in Z \text { for all } x \in \Lambda\right\}
$$

denote the polar lattice. It is well known that if $\Lambda=T \Gamma$, where $T$ is a nonsingular linear transformation and $\Gamma$ is the lattice of all points with integer coordinates, then $\Lambda^{*}=T^{*} \Gamma$, where $T^{*}$ is the inverse transpose of $T$. Let $F$ be a distance function for which the set

$$
C_{F}=\left\{x \in R^{n}: F(x)<1\right\}
$$

is a symmetric convex body. If $\mu$ denotes the volume of $C_{F}$, then Minkowski's convex body theorem states that there exists a point $x \neq 0$ of $\Lambda$ such that

$$
F(x) \leqslant 2 \mu^{-1 / n} d(\Lambda)^{1 / n} .
$$

Since $d\left(\Lambda^{*}\right) d(\Lambda)=1$ we therefore have the existence of nonzero points $x \in \Lambda$, $y \in \Lambda^{*}$ such that

$$
\mu^{2 / n} F(x) F(y) \leqslant 4 \text {. }
$$


For $n=2$ this result is best possible when $F\left(x_{1}, x_{2}\right)=\left|x_{1}\right|+\left|x_{2}\right|$ and $\Lambda$ is the lattice generated by $(1,0)$ and $(1 / 2,1 / 2)$. In this particular case we can in fact choose $x \in \Lambda, y \in \Lambda^{*}$ satisfying (1) and the further condition that they lie in the interior $P^{0}$ of the positive cone

$$
P=\left\{\left(x_{1}, x_{2}\right) \in R^{2}: x_{1} \geqslant 0, x_{2} \geqslant 0\right\} .
$$

One is therefore led to ask what can be said about $\mu^{2 / n} F(x) F(y)$ with $x, y$ nonzero points of $\Lambda, \Lambda^{*}$, respectively, when $x$ and $y$ are restricted to lie in either $P$ or $P^{0}$. A little trial and error suggests that $x \in P^{0}, y \in P^{0}$ is a little too strict a condition. $\dagger$ Considering $F\left(x_{1}, x_{2}\right)=\left|x_{1}\right|+\left|x_{2}\right|$ and $\Lambda$ generated by $(1,0)$ and $(-t, t)$, where $t$ is a positive integer, we have

$$
\mu F(x) F(y) \geqslant 2\left(t+t^{-1}+2\right)
$$

for all $x \in P^{0}, y \in P^{0}$, and so any upper bound on $\mu F(x) F(y)$ under these conditions would depend on both $F$ and $\Lambda$. The next condition to be considered would be $x \in P, y \in P^{0}$ (or, equivalently, $x \in P^{0}, y \in P$ ), and the aim of this paper is to give an upper bound depending only on $F$ for a couple of standard distance functions $F$. We show

THEOREM 1. Let $F_{t}$ denote the distance function on $R^{2}$ defined by

$$
F_{i}\left(x_{1}, x_{2}\right)=\left|x_{1}\right|+t\left|x_{2}\right|,
$$

where $t>0$, and let $\Lambda$ be a lattice in $R^{2}$. Then there exist nonzero $x \in P \cap \Lambda, y \in P^{0} \cap \Lambda^{*}$ such that

$$
\mu F_{l}(x) F_{l}(y) \leqslant 2\left(t+t^{-1}\right) .
$$

THEOREM 2. Let $G_{l}$ denote the distance function on $R^{2}$ defined by

$$
G_{l}\left(x_{1}, x_{2}\right)=\left(x_{1}^{2}+t^{2} x_{2}^{2}\right)^{\frac{1}{2}},
$$

where $t>0$, and let $\Lambda$ denote a lattice in $R^{2}$. Then there exist nonzero $x \in P \cap \Lambda$, $y \in P^{0} \cap \Lambda^{*}$ such that

$$
\mu G_{l}(x) G_{l}(y) \leqslant \pi\left(t^{2}+t^{-2}\right)^{\sharp} .
$$

Theorems 1 and 2 are best possible in that, for example, equality holds for any multiple of the lattice generated by $(1,0)$ and $\left(0, t^{-1}\right)$.

The applications of these theorems are to lattices $\Lambda$ containing $\Gamma$, for then $\Lambda^{*}$ consists entirely of integral points. For example, we have

† Henceforth $x$ will always stand for a non zero point of $\Lambda$ and $y$ a non zero point of $\Lambda^{*}$. 
COROLlaRY 1. Let $\Lambda$ be a lattice in $R^{2}$ containing $\Gamma$. Then there exists $\left(x_{1}, x_{2}\right) \in \Lambda \cap P$ and positive integers $l, m$ such that

$$
l x_{1}+m x_{2} \in Z
$$

and

$$
0<x_{1}+x_{2} \leqslant 2 /(l+m) .
$$

This is just the case $t=1$ of Theorem 1 , though (2) is weaker than the requirement that $\left(x_{1}, x_{2}\right)$ and $(l, m)$ lie in polar lattices.

COROLlaRY 2. Let $\theta_{1}, \theta_{2}$ be rationals. Then there exist positive integers $l, m$ such that $l \theta_{1}+m \theta_{2} \in Z$, and a positive integer $t$ such that

$$
0<\left\{t \theta_{1}\right\}+\left\{t \theta_{2}\right\} \leqslant 2 /(l+m),
$$

where $\{x\}$ denotes the fractional part of $x$.

This is just Corollary 1 applied to the lattice of all points of the form $\left(t \theta_{1}+a_{1}, t \theta_{2}+a_{2}\right)$ with $a_{1}, a_{2}$ integral. Of course using Theorem 2 in place of Theorem 1 would replace (3) by

$$
0<\left\{t \theta_{1}\right\}^{2}+\left\{t \theta_{2}\right\}^{2} \leqslant 2 /\left(l^{2}+m^{2}\right) .
$$

In addition, if $\|\theta x\|$ denotes $\min (\{x\}, 1-\{x\})$, then, since $\|t \theta\| \leqslant\{t \theta\}$ and $\|t \theta\|=0$ only when $\{t \theta\}=0$, we can clearly replace $\{t \theta\}$ by $\|t \theta\|$ in (3) and ( $\left.3^{\prime}\right)$.

It will be observed that Corollary 2 is best possible in some cases. For example, if $k$ is integral then when $\theta_{1}=\theta_{2}=k^{-1}$ and when $\theta_{1}=k^{-1}, \theta_{2}=1-k^{-1}$ we are dealing with lattices requiring equality in Theorem 1 (when $t=1$ ) and Corollary 2 .

The inequality (3) can be compared with the results for $\theta_{1}, \theta_{2}$ not necessarily rational, which are as follows.

Firstly, if $\theta_{1}, \theta_{2}, 1$ are independent over the rationals then the right-hand side of (3) can be replaced by $\varepsilon$ for any $\varepsilon>0$. A similar situation occurs when $\theta_{1}, \theta_{2}$ satisfy a relation of the type $l \theta_{1}-m \theta_{2}=n$ with $l, m, n$ positive integers. However, if $\theta_{1}, \theta_{2}$ satisfy a relation of the type $l \theta_{1}+m \theta_{2}=n$ with $l, m, n$ positive integers, then if $(l, m, n)=1$ the best that can be said is that the right-hand side of (3) can be replaced by $1 / \max (l, m)$.

Proof of THEOREM 1. The proof uses the fact that $(d, c) / d(\Lambda)$ lies in $\Lambda^{*}$ when $(-c, d) \in \Lambda$. Let $\alpha_{1}=(a, b) \neq(0,0)$ be a point of $\Lambda \cap P$ such that $a+b t$ is minimal and let $(-c, d)$ be a point of $\Lambda$ in the second quadrant

$$
Q=\left\{\left(x_{1}, x_{2}\right) \in R^{2}: x_{1}<0, x_{2}>0\right\}
$$


such that $c t+d$ is minimal. It is clear that

$$
\left\{\left(x_{1}, x_{2}\right) \in R^{2}:\left|x_{1}+t x_{2}\right|<a+b t,\left|t x_{1}-x_{2}\right|<c t+d\right\}
$$

contains no nonzero point of $\Lambda$, and so by Minkowski's linear forms theorem (Cassels (1959), p. 73) we have

$$
d(\Lambda) \geqslant(a+b t)(c t+d) /\left(1+t^{2}\right) .
$$

The theorem now follows immediately on setting $x=\alpha_{1}, y=(d, c) / d(\Lambda)$ and $\mu=2 t^{-1}$.

Proof of Theorem 2. The basic idea behind the proof of this theorem is the same as for the previous theorem, but Minkowski's theorem is not strong enough in this case. Let $x=(a, b) \neq(0,0)$ be a point of $\Lambda \cap P$ such that $A^{2}=a^{2}+t^{2} b^{2}$ is minimal, and let $(-c, d)$ be a point of $\Lambda \cap Q$ such that $B^{2}=t^{2} c^{2}+d^{2}$ is minimal. Since $y=(d, c) / d(\Lambda)$ lies in $\Lambda^{*} \cap P^{0}$, we only need to show

$$
t^{-1} A B \leqslant\left(t^{2}+t^{-2}\right)^{\frac{3}{2}} d(\Lambda)
$$

in order to prove the theorem. We assume without loss of generality that $a \neq 0$ and that $m \leqslant t$ where $b=a m$, for interchanging the roles of the coordinates replaces $m$ by $m^{-1}$ and $t$ by $t^{-1}$.

Since $x$ is a primitive lattice point there is a basis $\{x, \gamma\}$ of $\Lambda$, and replacing $\gamma$ by $-\gamma$ if necessary we may take $\gamma$ to lie on the line

$$
L=\left\{\left(x_{1}, x_{2}\right) \in R^{2}: x_{2}=m x_{1}+e\right\},
$$

where $e>0$. Furthermore, the lattice points $\gamma+n x$, for integral $n$, lie on $L$ at equally spaced intervals of length $l_{1}=a\left(1+m^{2}\right)^{\frac{1}{2}}$ and so $L$ cannot intersect

$$
T=\left\{\left(x_{1}, x_{2}\right) \in Q: t^{2} x_{1}^{2}+x_{2}^{2}<B^{2}\right\} \cup\left\{\left(x_{1}, x_{2}\right) \in P: x_{1}^{2}+t^{2} x_{2}^{2}<A^{2}\right\}
$$

in a continuous segment of length greater than $l_{1}$. We now establish the inequality

$$
B e^{-1} \leqslant\left\{\left(t^{4}+1\right) /\left(1+m^{2} t^{2}\right)\right\}^{1},
$$

which is equivalent to (4).

We note firstly that (5) is trivial if $B \leqslant e$ and so we assume $B>e$. We let

$$
\left.f=e\left\{m+\left[\left(B e^{-1}\right)^{2}\left(m^{2}+t^{2}\right)-t^{2}\right]\right\}\right\} /\left(m^{2}+t^{2}\right)
$$

and

$$
h=e\left\{\left[\left(A e^{-1}\right)^{2}\left(1+m^{2} t^{2}\right)-t^{2}\right]^{\frac{1}{3}}-m t^{2}\right\} /\left(1+m^{2} t^{2}\right) .
$$

Furthermore, we set $\sigma_{1}=(-f, e-m f), \sigma_{2}=\left(-e m^{-1}, 0\right), \rho_{1}=(0, e), \rho_{2}=(h, m h+e)$ and $e_{0}=A t^{-1}=a\left(m^{2}+t^{-2}\right)^{t}$.

Then $L$ meets $T$ in a continuous line segment, of length $l_{2}$, say, from the point $\sigma$, which is either $\sigma_{1}$ or $\sigma_{2}$, to the point $\rho$, where $\rho=\rho_{1}$ if $e \geqslant e_{0}$ and $\rho=\rho_{2}$ if $e<e_{0}$. 
We consider the various possibilities.

(i) If $e \geqslant e_{0}$ then $e m^{-1}>a$ and so $l_{2}>l_{1}$ if $\sigma=\sigma_{2}$. However, when $\sigma=\sigma_{1}$ we have

$$
l_{2} e^{-1}=f e^{-1}\left(1+m^{2}\right)^{\frac{1}{4}}
$$

which is an increasing funtcion of $B e^{-1}$. When

$$
B e^{-1}=\left[\left(t^{4}+1\right)\left(1+m^{2} t^{2}\right)\right]^{\sharp},
$$

we see that

$$
\begin{aligned}
l_{2} e^{-1} & =\left\{m\left(1+m^{2} t^{2}\right)^{\frac{1}{2}}+\left(t^{6}+m^{2}\right)^{\frac{1}{4}}\right\}\left(1+m^{2}\right)^{\frac{1}{4}}\left(m^{2}+t^{2}\right)^{-1}\left(1+m^{2} t^{2}\right)^{-t} \\
& >\left(m^{2} t+t^{3}\right)\left(1+m^{2}\right)^{\frac{1}{4}}\left(m^{2}+t^{2}\right)^{-1}\left(1+m^{2} t^{2}\right)^{-\frac{1}{t}} \\
& =t\left(1+m^{2}\right)^{\frac{1}{2}}\left(1+m^{2} t^{2}\right)^{-\frac{1}{4}}
\end{aligned}
$$

Hence if (5) is false we have, since $e \geqslant e_{0}$, that

$$
l_{2} \geqslant\left(l_{2} e^{-1}\right) e_{0}>t\left(1+m^{2}\right)^{ \pm}\left(1+m^{2} t^{2}\right)^{-\frac{t}{2}} e_{0}=l_{1} \text {, }
$$

which is impossible. Hence (5) must hold if $e \geqslant e_{0}$.

(ii) If $e<e_{0}$ we note that $l_{2} \leqslant l_{1}$ is equivalent to the inequality $f+h \leqslant a$. Since for fixed $e, f+h$ is an increasing function of $B$, (5) will follow on showing that $f+h \geqslant a$ when (6) holds. Suppose to the contrary that $f+h<a$ when (6) holds, that is,

$$
\begin{aligned}
\left\{m+\left[\left(t^{6}+m^{2}\right)\left(1+m^{2} t^{2}\right)\right]^{1}\right\} & \left(m^{2}+t^{2}\right)^{-1}-m t^{2}\left(1+m^{2} t^{2}\right)^{-1} \\
& <\omega-\left[\omega^{2}\left(1+m^{2} t^{2}\right)^{2}-t^{2}\right]^{\ddagger}\left(1+m^{2} t^{2}\right)^{-1},
\end{aligned}
$$

on writing $\omega$ for $a e^{-1}$ and re-arranging. The right hand side of this inequality decreases as $\omega$ increases, and $\omega>\left(m^{2}+t^{-2}\right)^{-1}$ and so (7) implies that

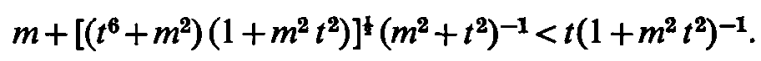

Since squaring this yields

$$
\left[\left(t^{6}+m^{2}\right)\left(1+m^{2} t^{2}\right)\right]^{\sharp}<m\left(t^{4}-1\right),
$$

which is obviously impossible, we have reached the desired contradiction, and our proof of Theorem 2 is complete.

\section{Reference}

J. W. S. Cassels (1959), An introduction to the geometry of numbers (Springer-Verlag, Berlin).

Department of Mathematics

University of Chittagong

Chittagong, Bangladesh
Department of Mathematics

Monash University

Victoria, Australia 3168 